\title{
Prolactinoma Presenting as Short-lasting Unilateral Neuralgiform Headache Attacks with Conjunctival Injection and Tearing Syndrome
}

Sir,

Short-lasting unilateral neuralgiform headache attacks with conjunctival injection and tearing (SUNCT) is usually a rare primary form of headache with prominent autonomic components and is grouped under trigeminal autonomic cephalalgia. ${ }^{[1]}$ Secondary causes of SUNCT due to intracranial pathology are usually seen in posterior cranial fossa lesions, but cases of pituitary adenoma causing this syndrome have also been described. ${ }^{[1]}$ Majority of cases reporting this association also reported a significant deterioration of headache with use of dopamine agonists (DA). However, we report a case of prolactinoma and coexistent SUNCT which resolved completely after cabergoline therapy.

Case description: A 22-year-old female presented with a headache of 10-year duration and secondary amenorrhea. She had 30-40 daily attacks of unilateral, stereotyped, excruciating stabbing pain in the right retrobulbar region radiating to temporal region associated with reddening and watering of the right eye [Figure 1a]. There was no history of associated nausea, vomiting photophobia, phonophobia, or aura. The attacks lasted for 15 days to 1 month. No specific triggering event was present. She reported that she had development of breast and pubic hair at an appropriate time. However, she attended menarche at 16 years of age and continued to have irregular periods for subsequent $1 \frac{1}{2}$ years. She has amenorrhea for the last 4 years. There was no family history of similar disorder. On evaluation, she was normotensive with a body mass index of $21.9 \mathrm{~kg} / \mathrm{m}^{2}$. Her sexual maturity rating status was P4 B5. Visual acuity and visual field were normal. There was galactorrhea on expression from both breasts. Rest general and systemic examination was normal. Except for the presence of headache, a detailed neurological evaluation revealed no specific abnormality. In view of galactorrhea and secondary amenorrhea, a serum prolactin assay was done. Serum prolactin level (with dilution) came out to be $2045 \mathrm{ng} / \mathrm{ml}$. Magnetic resonance imaging (MRI) of pituitary revealed a pituitary macroadenoma with suprasellar and parasellar extension [Figure 1b-e]. Pituitary hormone testing revealed low luteinizing hormone levels of $1.04 \mathrm{mIU} / \mathrm{ml}$ (normal 1.14-5.7 mIU/ml) and follicle-stimulating hormone of $1.6 \mathrm{mIU} / \mathrm{ml}$ (normal $1.34-13.5 \mathrm{mIU} / \mathrm{ml}$ ), respectively. Other pituitary hormones including basal cortisol (8 am), thyroid-stimulating hormone, free thyroxine, and insulin-like growth factor 1 were within normal range. Liver function tests, renal function tests, and serum electrolytes were normal. Based on above findings, a diagnosis of macroprolactinoma was made. She was started on cabergoline therapy $(0.5 \mathrm{mg}$ twice weekly). There was marked improvement in headache evident after 4 weeks of therapy and it completely disappeared at 3 months [Figure 1f]. There was disappearance of galactorrhea after 1 month of therapy. Serum prolactin normalized $(12 \mathrm{ng} / \mathrm{ml})$ by the end of $2^{\text {nd }}$ month and continued to remain so. She resumed menses by the end of 3 months of DA therapy. A follow-up pituitary MRI at 1 year showed gross reduction of tumor [Figure 1 $\mathrm{g}$ and $\mathrm{h}$ ].

SUNCT has been classified as a primary headache syndrome and is grouped under trigeminal autonomic cephalalgias (TAC) by Headache Classification Committee of the International Headache Society, 2013. ${ }^{[1]}$ These are stereotyped attacks of moderate or severe, strictly unilateral head pain on trigeminal nerve territory. To 


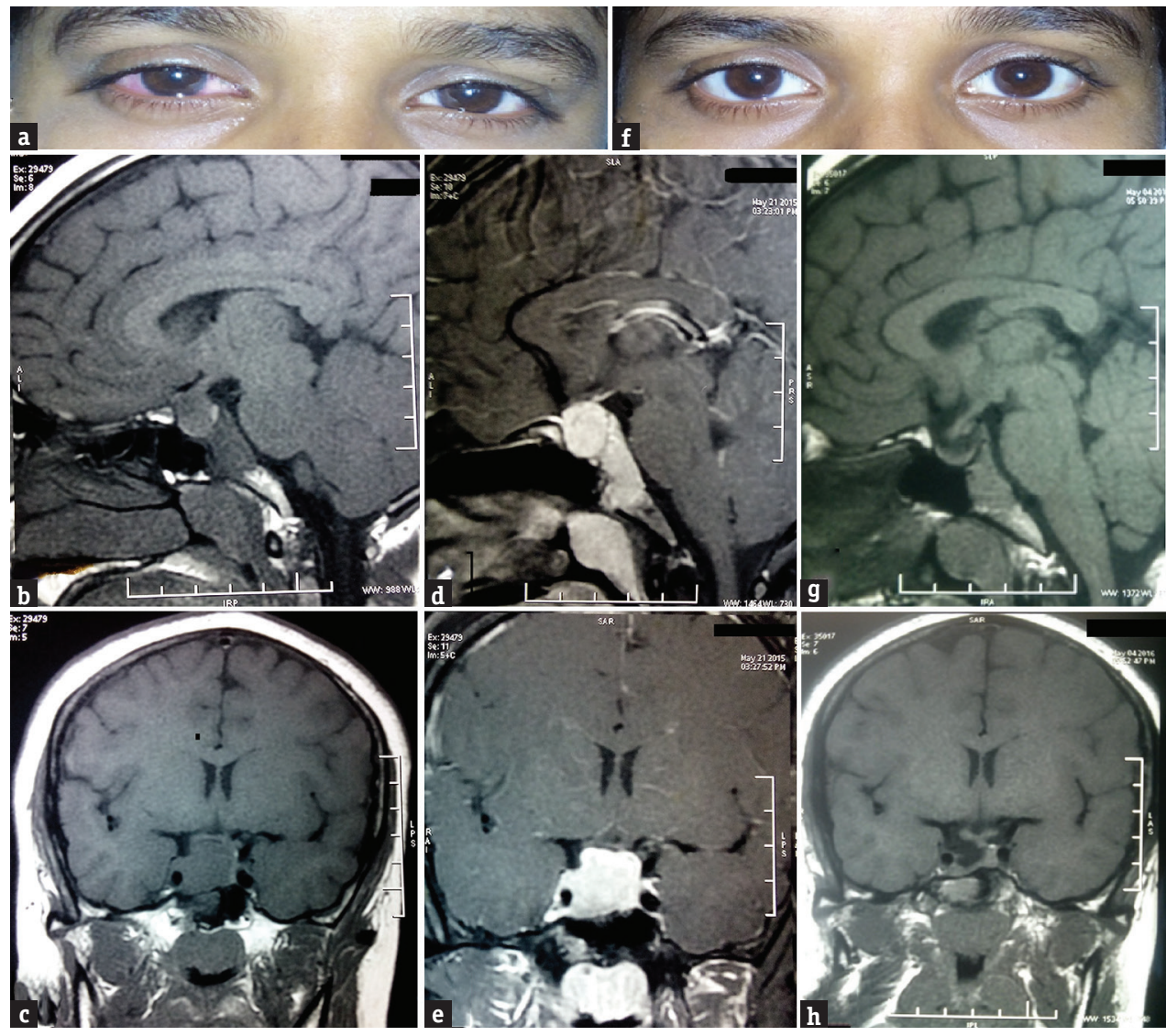

Figure 1: (a) Lacrimation and redness of right eye during headache. (b) T1-weighted image showing a macroadenoma $(1.8 \mathrm{~cm} \times 1.7 \mathrm{~cm} \times 2.3 \mathrm{~cm})$. (c) T1-weighted image showing macroadenoma causing stalk compression and parasellar extension. (d) Postcontrast image showing intense enhancement of macroadenoma. (e) Postcontrast image showing intense enhancement of macroadenoma. (f) Recovery after drug treatment. (g) T1-weighted image showing a significant tumor size reduction after treatment. (h) T1-weighted image showing significant tumor size reduction after treatment

define SUNCT at least twenty attacks of moderate or severe unilateral head pain, with orbital, supraorbital, temporal, and/or other trigeminal distribution, lasting for $1-600 \mathrm{~s}$ and occurring as single stabs, series of stabs, or in a sawtooth pattern with the presence of at least one of the following cranial autonomic symptoms or signs, ipsilateral to the pain is to be present: conjunctival injection and/or lacrimation, nasal congestion and/or rhinorrhea, eyelid edema, forehead and facial sweating, forehead and facial flushing, sensation of fullness in the ear miosis and/or ptosis. ${ }^{[1]}$ SUNCT is a rare primary headache syndrome but may be manifested usually secondary to posterior cranial fossa lesions. ${ }^{[1]}$ Conditions associated with SUNCT manifesting as secondary headache include pituitary microadenomas and macroadenomas, posterior fossa lesions, cerebral infarction, meningitis and encephalitis, neuroinflammatory disorders, temporomandibular joint disorders, and vascular anomalies such as loops and dural fistulas. ${ }^{[2]}$

A large proportion of patients with pituitary adenomas (37.5\%-70\%) suffer from headache, primarily presenting as migraine, or tension-type headache. The much less frequently reported TAC, may, however, constitute a true secondary headache type associated with pituitary adenomas. ${ }^{[3]}$ Headache is the common and disabling symptoms experienced by people harboring pituitary adenoma with a marked clinical heterogeneity, a wide variety of headache types, including SUNCT has been reported. ${ }^{[4]}$ The pathophysiology of the headache associated with pituitary tumors is not completely clear. Dural stretch, invasion of the cavernous sinus, and local pressure effects have been suggested as mechanisms..$^{[3,4]}$ It has been found that differences in tumor size were not apparent between those who presented headaches and those who did not. ${ }^{[4]}$ Furthermore, there were no clear correlations between the pituitary volume and headache score. ${ }^{[4]}$ The extent of cavernous sinus invasion was not associated with the presence/extent of headache. ${ }^{[4]}$

As mechanical factors fail to completely explain the association of headache with pituitary adenomas, a dysregulation of hypothalamic neurohormonal factors has been to put forth as a possible explanation. Dysfunction of the dopamine prolactin axis, growth hormone (GH), and the trigeminovascular system plays a causative role. ${ }^{[4]}$ The majority of the pituitary tumors causing SUNCT are either prolactinoma or GH-secreting adenomas and removal of 
these tumors can be associated with clinical remission. ${ }^{[4]}$ Histological expression of none of the biochemical factors investigated so far such as neuropeptide $\mathrm{Y}$, calcitonin gene-related peptide, substance $\mathrm{P}$, or vasoactive intestinal polypeptide was related to headache in patients with pituitary adenomas. ${ }^{[3]}$ SUNCT can be associated with both pituitary microadenomas ${ }^{[5-9]}$ and macroadenomas. ${ }^{[2,4,8,10,11]}$ A vast majority of reported cases had prolactinoma, ${ }^{[4,6,8-12]}$ whereas few had GH-secreting tumors ${ }^{[4,5,7]}$ and nonfunctioning adenomas. ${ }^{[2,8]}$ Hence, there is a strong association of these headaches with prolactinomas and it seems plausible that dopamine-prolactin axis plays a predominant role in such cases.

The relationship between DA and SUNCT deserves special mention. It is quite complex and paradoxical to say the least. Majority of reported cases had induction or dramatic deterioration of headache with institution of DA therapy. ${ }^{[4,6,8,9,12,13]}$ It could be attributed to role of central dopamine-prolactin axis. This poses an important clinical challenge in such cases as DA may have to be stopped if significant worsening of headache occurs. It is a well-known fact that prolactinomas have an excellent response to DA therapy, but in association with SUNCT, other modalities of therapy may have to be explored. Hence, it is important for endocrinologists to be aware of such association. However, quite contrary to above cases, rare instances of complete remission of headache with DA therapy as seen in our case have also been reported. ${ }^{[10,11]}$ A common finding among these cases including ours was that all had macroadenomas. Therefore, it may be possible that reduction in tumor size could have resulted in improvement of symptoms. The reduction of tumor size in large prolactinomas may improve headache through structural changes although there is a little evidence for the size of the tumor being generally important. ${ }^{[3]}$ The DA which have been reported to induce SUNCT attacks in patients with prolactinoma include both ergot and nonergot derivatives such as bromocriptine, ${ }^{[6,13]}$ lisuride, ${ }^{[13]}$ quinagolide, ${ }^{[4,6]}$ and cabergoline. ${ }^{[6,13]} \mathrm{DA}$ share properties with ergot alkaloids and ergot alkaloids are known to alter the activity of the trigeminovascular system. ${ }^{[4]}$ The role of surgery resulting in clinical remission in few cases has also been described. ${ }^{[5-8]}$ Recently, a radiation-induced SUNCT case has been reported. ${ }^{[2]}$

Based on case reports, lamotrigine appears to be the most effective medication for SUNCT. Other treatment options include gabapentin, topiramate, verapamil, carbamazepine, zonisamide, intravenous lidocaine, intravenous phenytoin, and greater occipital nerve block. $^{[8]}$ As a prophylactic medication, lamotrigine has been reported to show a response rate in SUNCT of $68 \%-100 \%$ and topiramate a response of $52 \% \cdot{ }^{[8]}$ Identifying SUNCT early is pivotal not only for therapeutic purpose but also for a thorough search for potential causes such as pituitary adenoma. It has been rarely seen that such headache can predate other endocrine or neurological symptoms by years. ${ }^{[9]}$

As exemplified by our case, pituitary adenoma may manifest initially with confusing nonspecific symptoms such as SUNCT years before the eventual diagnosis is made due to hormonal abnormalities. A high degree of clinical suspicion is essential for early diagnosis of such cases. Identifying SUNCT at an early state could prompt the physician to search for secondary organic causes such as pituitary adenoma. On the other hand, endocrinologists who routinely prescribe DA for treatment of prolactinomas and idiopathic hyperprolactinemia should have knowledge about SUNCT associated with DA therapy. Although SUNCT associated with prolactinomas deteriorate with DA therapy, some cases may respond well.

\section{Financial support and sponsorship}

Nil.

\section{Conflicts of interest}

There are no conflicts of interest.

Swayamsidha Mangaraj, Pratap Kumar Mishra, Arun Kumar Choudhury, Binoy Kumar Mohanty, Anoj Kumar Baliarsinha

Department of Endocrinology, S.C.B Medical College, Cuttack, Odisha, India

Address for correspondence: Dr. Swayamsidha Mangaraj, Department of Endocrinology, S.C.B Medical College, Cuttack - 753 007, Odisha, India. E-mail: drsmangaraj@gmail.com

\section{REFERENCES}

1. Headache Classification Committee of the International Headache Society (IHS). The International Classification of Headache Disorders, $3^{\text {rd }}$ edition (beta version). Cephalalgia 2013;33:629-808.

2. Berk T, Silberstein S. Case report: Secondary SUNCT after radiation therapy - A novel presentation. Headache 2016;56:397-401.

3. Kreitschmann-Andermahr I, Siegel S, Weber Carneiro R, Maubach JM, Harbeck B, Brabant G. Headache and pituitary disease: A systematic review. Clin Endocrinol (Oxf) 2013;79:760-9.

4. Levy MJ, Matharu MS, Meeran K, Powell M, Goadsby PJ. The clinical characteristics of headache in patients with pituitary tumours. Brain 2005;128(Pt 8):1921-30.

5. Rozen TD. Resolution of SUNCT after removal of a pituitary adenoma in mild acromegaly. Neurology 2006;67:724.

6. Levy MJ, Matharu MS, Goadsby PJ. Prolactinomas, dopamine agonists and headache: Two case reports. Eur J Neurol 2003;10:169-73.

7. Adamo MA, Drazin D, Popp AJ. Short-lasting, unilateral neuralgiform headache attacks with conjunctival injection and tearing syndrome treated successfully with transsphenoidal resection of a growth hormone-secreting pituitary adenoma. J Neurosurg 2008;109:123-5. 
8. Chitsantikul P, Becker WJ. SUNCT, SUNA and pituitary tumors: Clinical characteristics and treatment. Cephalalgia 2013;33:160-70.

9. Larner AJ. Headache induced by dopamine agonists prescribed for prolactinoma: Think SUNCT! Int J Clin Pract 2006;60:360-1.

10. Matharu MS, Levy MJ, Merry RT, Goadsby PJ. SUNCT syndrome secondary to prolactinoma. J Neurol Neurosurg Psychiatry 2003;74:1590-2.

11. Kallestrup MM, Kasch H, Østerby T, Nielsen E, Jensen TS, Jørgensen JO. Prolactinoma-associated headache and dopamine agonist treatment. Cephalalgia 2014;34:493-502.

12. Massiou H, Launay JM, Levy C, El Amrani M, Emperauger B, Bousser MG. SUNCT syndrome in two patients with prolactinomas and bromocriptine-induced attacks. Neurology 2002;58:1698-9.

13. Jiménez Caballero PE. SUNCT syndrome in a patient with prolactinoma and cabergoline-induced attacks. Cephalalgia 2007;27:76-8.
This is an open access article distributed under the terms of the Creative Commons Attribution-NonCommercial-ShareAlike 3.0 License, which allows others to remix, tweak, and build upon the work non-commercially, as long as the author is credited and the new creations are licensed under the identical terms.

\begin{tabular}{|l|l|}
\hline \multicolumn{2}{|c|}{ Access this article online } \\
\hline Quick Response Code: & Website: \\
& \\
&
\end{tabular}

How to cite this article: Mangaraj S, Mishra PK, Choudhury AK, Mohanty BK, Baliarsinha AK. Prolactinoma presenting as short-lasting unilateral neuralgiform headache attacks with conjunctival injection and tearing syndrome. J Neurosci Rural Pract 2017;8:S158-61.

(C) 2017 Journal of Neurosciences in Rural Practice | Published by Wolters Kluwer - Medknow 